\title{
Tilting Operations for Small Screen Interfaces (Tech Note)
}

\author{
Jun Rekimoto \\ Sony Computer Science Laboratory Inc. \\ 3-14-13 Higashi-Gotanda, Shinagawa-ku, \\ Tokyo 141, Japan \\ rekimoto@csl.sony.co.jp \\ http://www.csl.sony.co.jp/person/rekimoto.html
}

\begin{abstract}
This TechNote introduces a novel interaction technique for small screen devices such as palmtop computers or hand-held electric devices, including pagers and cellular phones. Our proposed method uses the tilt of the device itself as input. Using both tilt and buttons, it is possible to build several interaction techniques ranging from menus and scroll bars, to more complicated examples such as a map browsing system and a 3D object viewer. During operation, only one hand is required to both hold and control the device. This feature is especially useful for field workers.
\end{abstract}

KEYWORDS: small screen interfaces, interaction techniques, palmtop computers

\section{INTRODUCTION}

The most significant difference between desktop computer$\mathrm{s}$ and hand-held computers is not the computation power, but the size of the screen. It is often impractical to simply apply the user interface for desktop computers to palmtop sized computers. For example, the desktop metaphor and the multiple windows metaphor are not so effective for the small screen of a Personal Digital Assistant (PDA). Many PDAs use a different user-interface metaphors, such as the notebook or cardfile metaphor.

Another major difference between PCs and small-screen devices is the input method. Pens and touch panels are often used for PDAs, but there are several disadvantages. These interfaces require two hands for operation (one for holding the display unit and the other for holding the pen). Pens tend to get lost and the pen hides the screen area. With very small electronic devices such as a pager, it is near impossible to use a pen interface due to its limited screen size.

\section{TILTING AS INPUT}

Instead of using a pen or a touch sensitive display, there are other possibilities to operate hand-held devices. The idea of using positions and orientations of palmtop computers as input was first introduced by the Chameleon system [2]. In this paper, we explore the idea of using tilt as the input method.
Sensing rotation of the device is much easier than sensing motions, by using small solid-state angular rate sensors (gyros). We can also use an electric inclinometer or a compass to assist the other sensors. As these types of sensors become used in other devices, we believe that the tilting interface becomes much more practical. Unlike pen interfaces, tilting allows single hand operation (i.e., only one hand is required to hold and operate the device). This feature is particularly useful for very small electronic devices such as pagers.

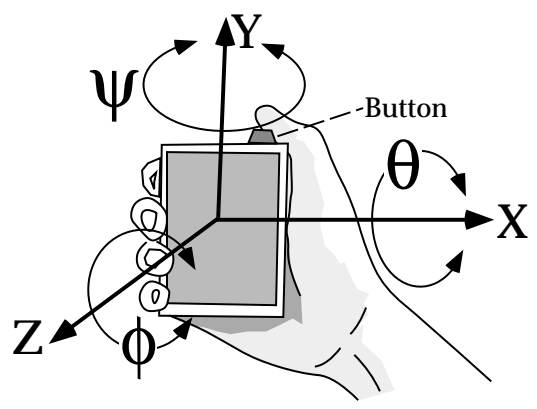

Figure 1: The operational concept of the tilting interface
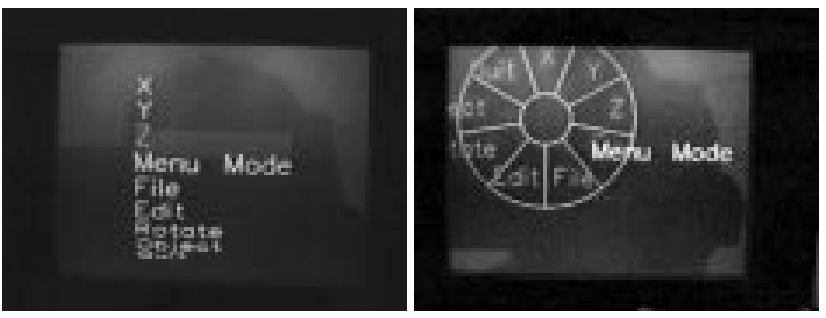

Figure 2: Two variations of tilting menus

\section{THE PROTOTYPE SYSTEM}

To thoroughly investigate the tilting interface, we built a prototype system consisting of a palmtop display (an LCD TV), a FASTRAK position and orientation sensor, and two buttons. The back-end workstation (SGI Indigo2) takes input from the sensor and the buttons through serial cables, and generates corresponding screen images for the display. The FASTRAK reports the absolute orientation of the sensor in the world coordinates, and the system converts it into the relative rotation about the body coordinate system $(\theta, \psi$, and $\phi$ in Figure 1). 


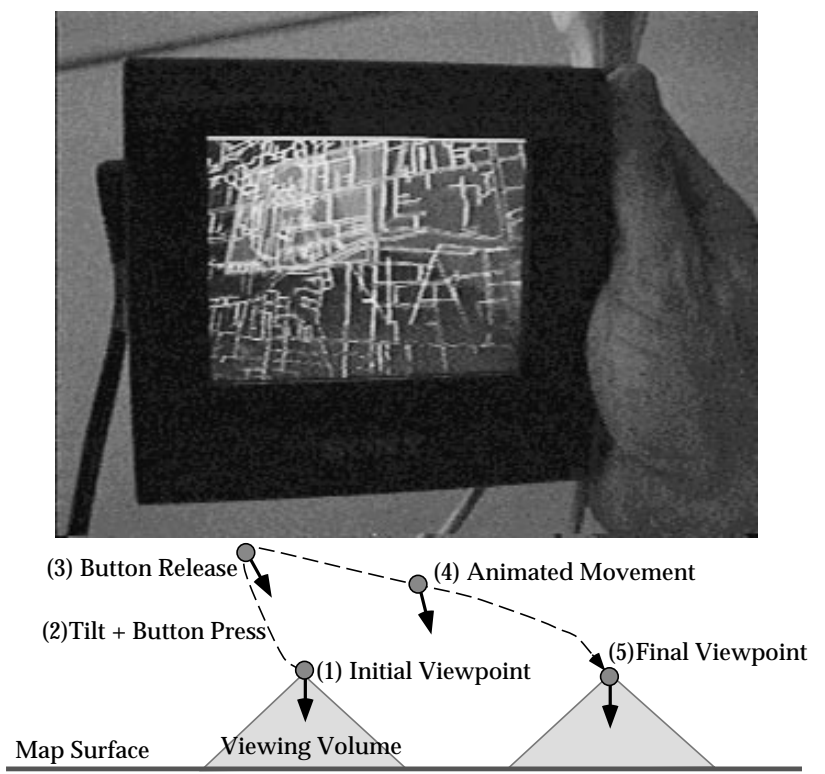

Figure 3: Navigating a map by tilting the display

\section{TILT MENUs}

The first example is a menu interface. We implemented two variations, the cylindrical menu and the pie menu (Figure 2). The basic interactions are the same; a user first presses the button and moves a menu item to the center of the screen by tilting the display while keeping the button depressed, then releases the button. The cylindrical menu is a $1 \mathrm{D}$ menu and users select an item by rotating the display about the horizontal axis. Menu items are placed on the cylinder surface to make a selected item larger while displaying all items. We adapted an already developed pie menu [1] to our tilting interface. Tilting scroll-bars can also be implemented.

An informal evaluation suggested that users could control the tilt quite precisely if visual feedback was adequately designed and provided. With the cylindrical menu, for example, the user was able to control the menu selection from one item to the next with only a 2 degree tilt. Normally, the user normally tilts the display 10-15 degrees during operation. Within this angle, the visibility of the LCD screen is not affected by the tilting.

There are several alternatives on menu designs. Our current implementation maps rotation about the vertical axis to horizontal movement of the menu items, and rotation about the horizontal axis to vertical movement. Instead of moving menu items, it could also be possible to control a cursor by tilting while menu items are fixed on the screen. This is much like a typical mouse interface, but we selected the cursor-fixed \& menu-floating approach because it allows larger menu items than the screen size. This would be a desirable feature for small screens that have a restricted screen resolution and size.

\section{NAVIGATING A LARGE 2D SPACE}

The second example is a map browser. Generally, the entire area of a map is too large to fit within a small screen, but simple scrolling interfaces are often frustrating. Therefore, we need a quick and intuitive navigation technique for effective viewing. Our solution is to provide a perspective view of the map, and allow the user to control the viewpoint by tilting the display. Unlike other proposed methods to achieve "focus and context" [3], our system does not use nonlinear projection, but simply relies on perspective projection. When a user wishes to view the right side of a map, for example, the user presses the button and tilts the display toward the right. The system shows a map from a viewpoint which is located left-hand side of the current viewpoint. At the same time, a rectangle cursor representing the target position appears on the surface of the map. The user controls the location of the cursor and looks around the map by keeping button depressed and rotating the display left and right, or up and down (Figure 3). When the user released the button, the viewpoint smoothly approaches to the designated position and the user sees animated movement toward the destination.

This interface is more than just a 3D visualization of the map. When a user tilts the display, the following transformation is applied to the map surface for visualization (the map is placed on the $\mathrm{X}-\mathrm{Y}$ plane and the viewpoint is looking into the negative $\mathrm{Z}$ axis):

$$
\begin{aligned}
& \operatorname{Trans}\left(C_{x}, C_{y},-A_{1} \max (|\psi|,|\theta|)\right) * \\
& \operatorname{Rot}\left(A_{2} \theta, A_{2} \psi, 0\right) * \operatorname{Trans}\left(-C_{x},-C_{y}, 0\right)
\end{aligned}
$$

where $\operatorname{Trans}(x, y, z)$ is a translation, $\operatorname{Rot}(\theta, \psi, \phi)$ is a rotation at an angle of $\theta$ about the $X$ axis, $\psi$ about the $\mathrm{Y}$ axis, and $\phi$ about the $\mathrm{Z}$ axis. $\left(C_{x}, C_{y}\right)$ is the center of rotation, and $A_{1,2}$ are constants. This equation simultaneously controls the three effects of viewing transformation (panning, zooming, and rotating) in an interrelated manner. The actual viewpoint movement is shown in Figure 3.

\section{D OBJECT INSPECTION}

We also built a 3D object viewer. In this example, the user inspects a 3D object model using a palmtop display. While pressing the button, the orientation of the $3 \mathrm{D}$ object is coupled to the orientation of the display so that it looks as if the object were fixed to the frame of the screen. Users can see 3D models from different directions just like inspecting a small object in their hand.

\section{CONCLUSION}

We have presented a new interaction technique that takes the tilt of a small display as input commands. This interface is being used and evaluated by our hand-held augmented reality device (HARD) project. Future plans include integration with other interaction techniques (e.g., tilt marking menus), and more human-factor evaluations (e.g., how Fits' law applies to tilting operations).

\section{ACKNOWLEDGMENTS}

I thank Toshiyuki Masui for helpful discussions.

\section{REFERENCES}

1. Jack Callahan, Don Hopkins, Mark Weiser, and Ben Shneiderman. An empirical comparison of pie vs. linear menus. In CHI'88, pages 95-100, 1988.

2. George W. Fitzmaurice, Shumin Zhai, and Mark H. Chignell. Virtual Reality for palmtop computers. ACM Transactions on Information Systems, 11(3):197-218, July 1993.

3. George Furnas. Generalized fisheye views. In $\mathrm{CHI}$ '86, pages 16-23, 1986. 